\title{
Dysembryoplastic neuroepithelial tumor and oligodendroglioma: the diagnostic value of magnetic resonance spectroscopy
}

\section{Case report and review of the literature}

Eric W. Sherburn, M.D., Mark M. Bahn, M.D., Ph.D., Murat Gokden, M.D., Daniel L. Silbergeld, M.D., and Keith M. Rich, M.D.

Department of Neurosurgery, Mallinckrodt Institute of Radiology and Division of Neuropathology, Washington University School of Medicine, St. Louis, Missouri; Department of Neurosurgery, University of Washington, Seattle, Washington

Preoperative differentiation between dysembryoplastic neuroepithelial tumor (DNT) and low-grade glioma is often not possible. Dysembryoplastic neuroepithelial tumor is a recently described entity of uncertain origin; however, the diagnosis has important clinical implications. Clinical and radiological findings of DNT and low-grade glioma, especially oligodendroglioma, may be similar. Treatment options and prognosis differ significantly between these two lesions; consequently, accurate diagnosis is imperative. The authors describe two individuals who presented simultaneously at their institution: one patient with an oligodendroglioma and a second patient with DNT. The natural history, neurodiagnostic, and pathological features of each are reviewed with special emphasis on the potential utility of magnetic resonance spectroscopy in differentiating these lesions.

Key Words * brain tumor * glioma * magnetic resonance imaging * seizures * surgery

The distinction between dysembryoplastic neuroepithelial tumor (DNT) and low-grade glioma, especially oligodendroglioma, may be a difficult one to make. Dumas-Duport, et al.,[7] who coined the term "dysembryoplastic neuroepithelial tumor," recently described 39 cases of supratentorial lesions representing this previously undescribed pathological entity. Although the authors believed these lesions represented neoplasms, the high incidence of cortical dysplasia suggests an embryonal origin. Since the recognition of this entity, several case reports and small series have been published.[1,7-10,14,15,17,25,26,28] Typically, patients with DNT present before the third decade of life with intractable complex partial seizures, with minimal, if any, neurological deficit. Treatment of DNT with surgical resection is "curative." It is important that DNT and glioma are correctly distinguished at diagnosis, because patients with DNT need not be subjected to potentially harmful adjuvant therapies such as radiation or chemotherapy.

Differentiating DNTs from low-grade gliomas, especially oligodendrogliomas, may be difficult, if not impossible, clinically and radiographically. Additionally, these lesions may be difficult to distinguish pathologically because they share similar histological features. This problem is compounded further by the widespread use of needle biopsy which, due to small sample size, may make accurate histopathological 
diagnosis difficult. We present two patients who presented simultaneously to our institution: one with an oligodendroglioma and another with a DNT. The clinicopathological differences between these two entities are emphasized. We also describe the potential value of magnetic resonance (MR) spectroscopy in differentiating DNTs from oligodendrogliomas.

\section{CASE REPORTS}

\section{Case 1}

This patient is a 40-year-old woman who suffered a generalized tonic-clonic seizure. The patient presented to the emergency department for evaluation. Neurological examination was normal. She underwent MR imaging with and without intravenous administration of gadolinium that revealed a left frontal mass (Fig. 1).
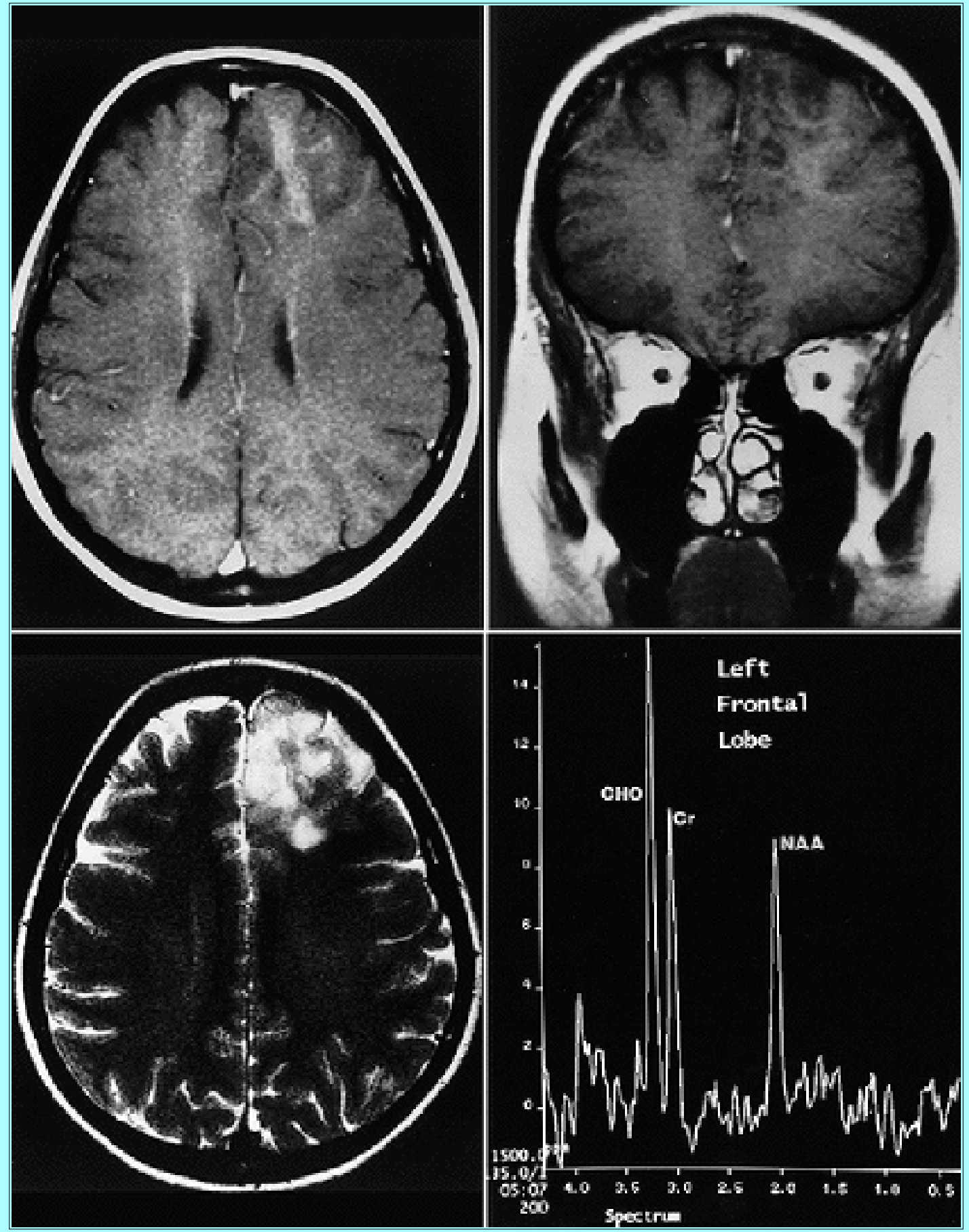

Fig. 1. Case 1. Neuroimaging studies demonstrating an oligodendroglioma. Upper Left: Axial 
$\mathrm{T}_{1}$-weighted gadolinium-enhanced MR image demonstrating a left frontal lobe mass with effacement of sulci and mild enhancement. Upper Right: Coronal $\mathrm{T}_{1}$-weighted gadolinium-enhanced MR image. Lower Left: Axial $\mathrm{T}_{2}$-weighted MR image demonstrating left frontal lobe mass with sulcal effacement and mild edema. Lower Right: An MR spectroscopy spectrum of the left frontal mass demonstrating decreased NAA compared to choline (CHO). The ratio of creatine $(\mathrm{Cr})$ to $\mathrm{CHO}$ is also reversed.

The lesion was thought to represent a low-grade glioma or a DNT. Magnetic resonance spectroscopy was performed to characterize the mass further, which demonstrated suppression of the $\mathrm{N}$-acetyl-aspartate (NAA) and creatine/phosphocreatine peaks with an increase in the choline peak suggestive of a primary neoplasm. The patient underwent elective craniotomy in which intraoperative stereotactic navigation was used and the frontal lobe mass was resected. Pathological evaluation of the surgical specimen revealed a World Health Organization[11] Grade II-IV oligodendroglioma (Fig. 2).

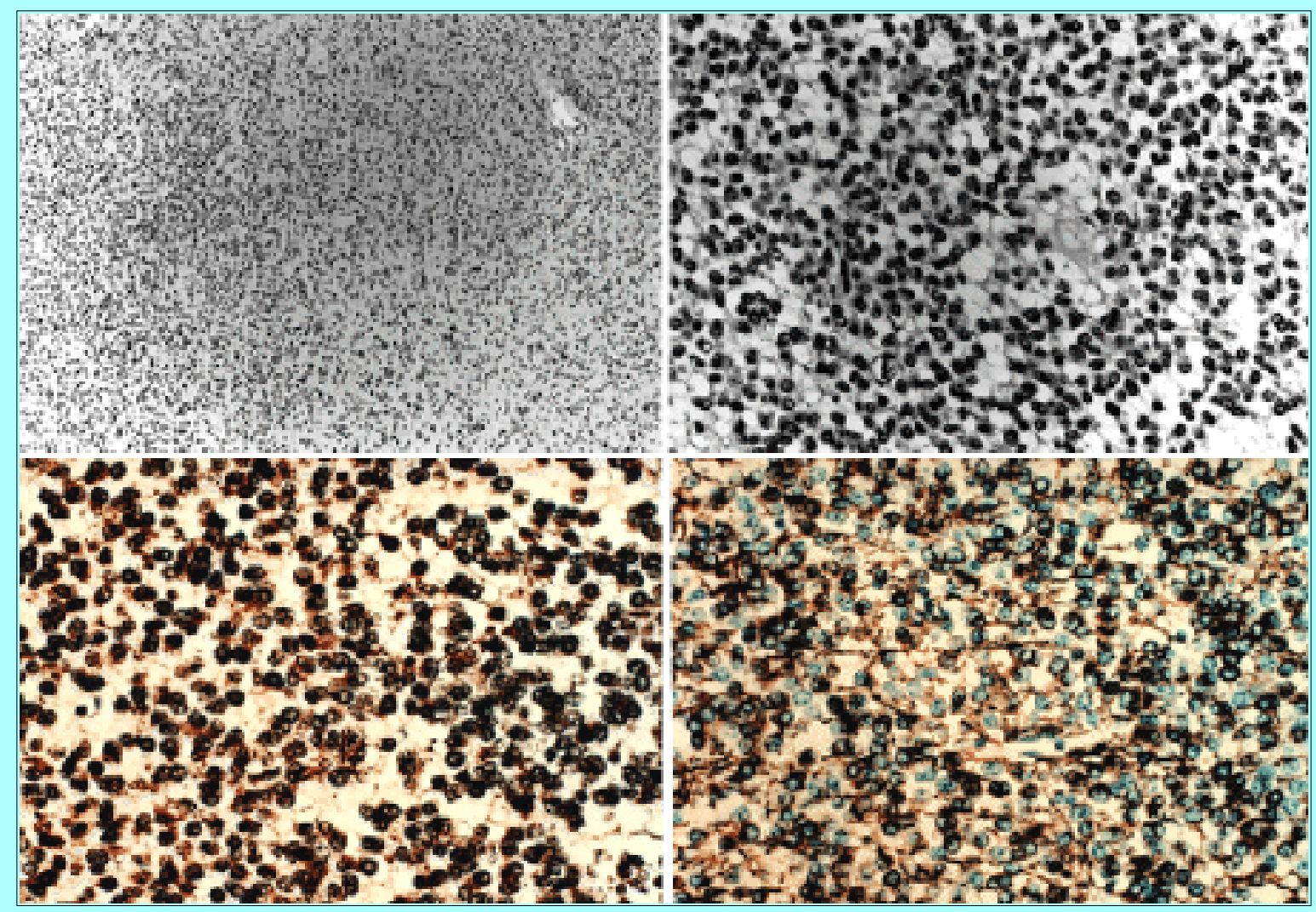

Fig. 2. Case 1. Photomicrographs showing the histological and immunohistochemical features of an oligodendroglioma. Upper Left: Infiltrative margin of the oligodendroglioma is demonstrated with the hypercellular area to the left representing bulk tumor. The cellularity gradually decreases to the right. Perineuronal satellitosis is also seen. $\mathrm{H} \& \mathrm{E}$, original magnification X 10. Upper Right: High-power view of the oligodendroglioma demonstrating sheets of uniform cells. Focal microcyst formation is also present. H \& E, original magnification X 200. Lower Left: Strong GFAP staining in this oligodendroglioma reveals minigemistocytic features. Original magnification X 200. Lower Right: Oligodendroglioma demonstrating strong S-100 protein positivity. Original magnification X 200.

\section{Case 2:}

This 19-year-old man had a history of complex partial seizures since the age of 12 years. His seizure disorder had become refractory to medical management, including treatment with phenytoin, phenobarbital, 
carbamazepine, and gabapentin. The patient was referred to our institution for evaluation and possible surgical treatment of his seizure disorder. His neurological examination, including visual field testing, was normal. He underwent MR imaging with and without intravenous administration of gadolinium that demonstrated a right occipital lobe mass (Fig. 3). The lesion was further characterized by using MR spectroscopy, which showed a spectral array pattern that is indistinguishable from normal brain. A 18fluorodeoxyglucose-positron emission tomography scan was performed, revealing hypometabolism in the region of the mass.
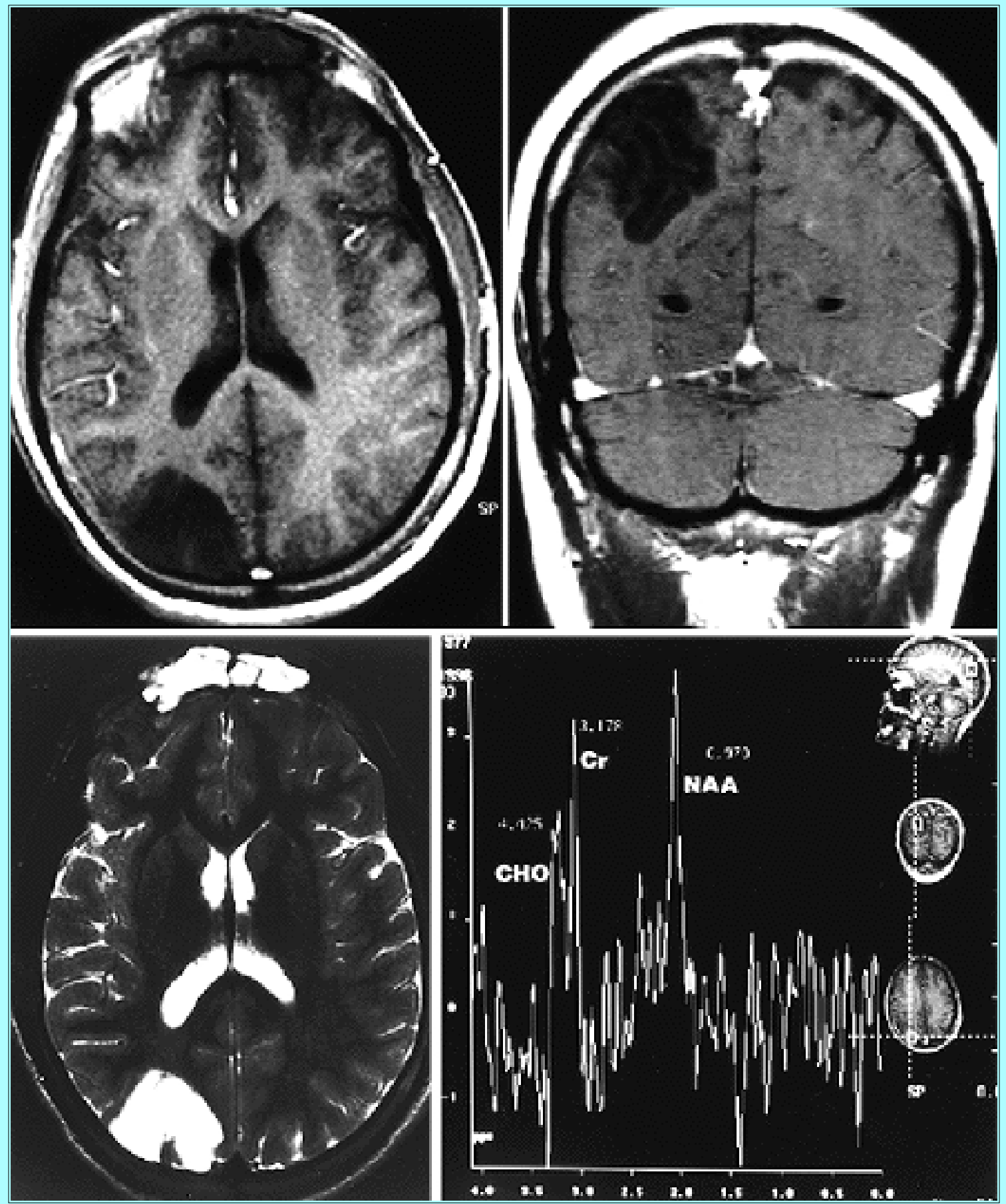

Fig. 3. Case 2. Neuroimaging studies demonstrating a DNT. Upper Left: Axial $\mathrm{T}_{1}$-weighted gadolinium-enhanced MR image demonstrating a hypointense right parietal lobe mass without mass effect or contrast enhancement. Upper Right: Postcontrast coronal $\mathrm{T}_{1}$-weighted MR image of the right parietal mass. Lower Left: Axial $\mathrm{T}_{2}$-weighted $\mathrm{MR}$ image demonstrating a hyperintense right parietal mass without mass effect or edema. Lower Right: An MR 
spectroscopy spectrum of the right parietal mass. This array reveals normal NAA, choline $(\mathrm{CHO})$, and creatine $(\mathrm{Cr})$ peaks resembling normal brain.

The patient underwent a left parietal craniotomy for placement of subdural and depth electrodes for electrocorticography and ictal mapping; intraictal mapping revealed seizure onset in the area surrounding the lesion but not from within the lesion itself. Subsequently, the patient underwent a second craniotomy for resection of the lesion, and a topectomy of the epileptogenic foci was performed. Pathological examination of the surgical specimen revealed it to be a DNT (Fig. 4).

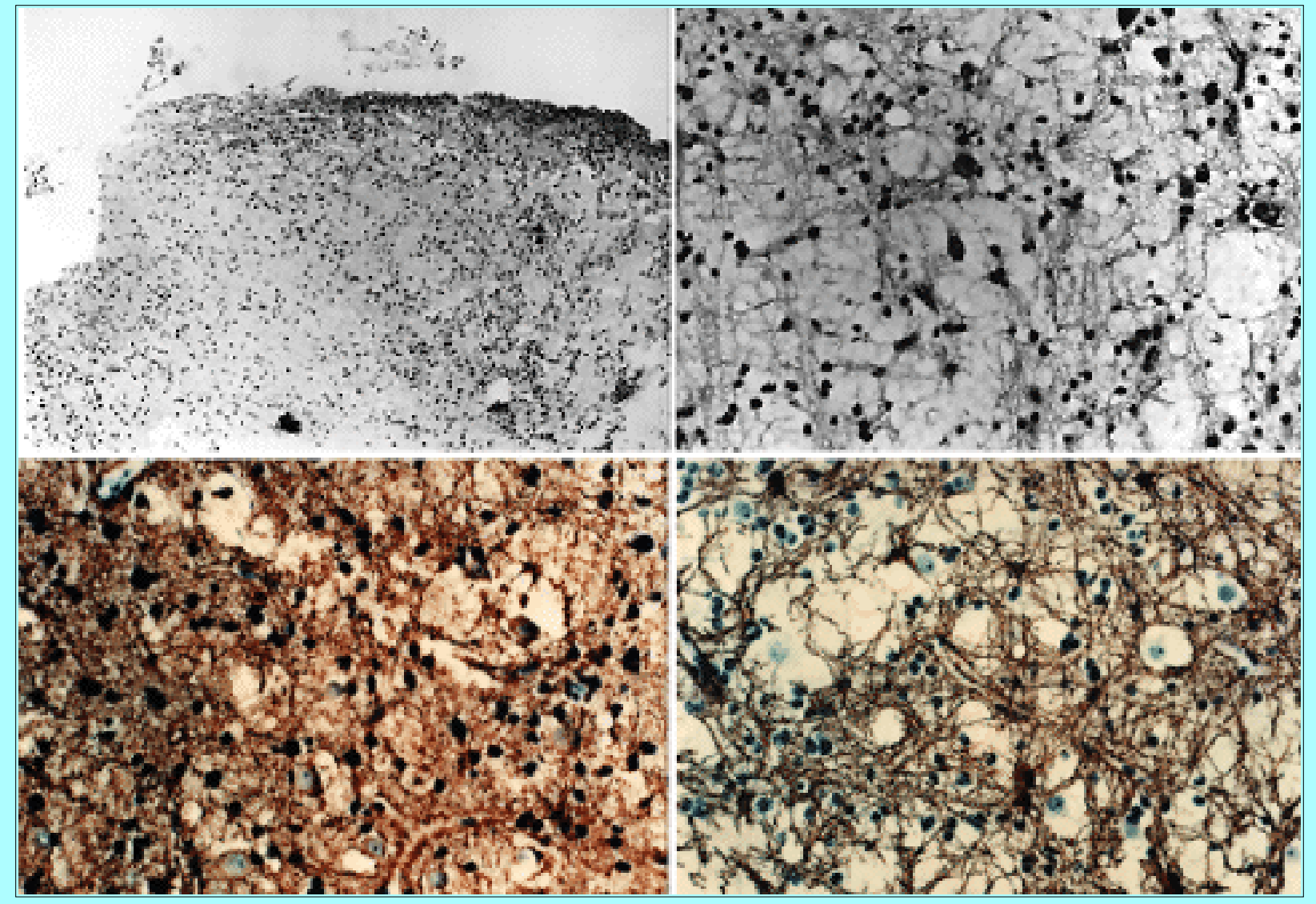

Fig. 4. Case 2. Photomicrographs showing the histological and immunohistochemical features of DNT. Upper Left: A distinct margin between the DNT (right) and underlying brain (left) is seen. The adjacent brain to the left is mildly gliotic (low power). $\mathrm{H} \& \mathrm{E}$, original magnification X 10. Upper Right: High-power view of the DNT demonstrating a disorganized admixture of glia and neurons in a mucin-rich background. H \& E, original magnification X 200. Lower Left: Staining with GFAP in a DNT demonstrating the glial component of the glioneuronal element. Original magnification X 200. Lower Right: Glial cells stained positive for S-100 protein in DNT. Note that the neurons appear to be free floating within the mucinous background. Original magnification X 200.

\section{DISCUSSION}

\section{Clinical Presentation}

Oligodendroglioma are relatively uncommon glial neoplasms that account for 4 to $5 \%$ of all intracranial primary neoplasms.[21] These tumors are most commonly diagnosed in the fourth decade of life.[4,21,22,30] Only $6 \%$ of all oligodendroglioma are diagnosed in the pediatric population, with the majority of these occurring in the second decade of life.[21,30] A male preponderance is reported in most large series[21,22,30] and may be as high as 2:1.[32] The most common presenting feature is seizures, which are 
found in more than $50 \%$ of cases $[4,21,22,32]$ and up to $88 \%$ of patients will have at least one seizure during the course of their illness.[30] The types of seizures exhibited vary between partial, generalized, or both.[32] Headache or increased intracranial pressure has been found in 20 to $30 \%$ of patients at presentation, $[4,22,32]$ and focal neurological deficits may be found in 13 to $35 \%$ of patients at the time of diagnosis.[4,22,32] Interestingly, in patients with oligodendroglioma, there may be a long delay between the onset of symptoms and the time of diagnosis, with a mean duration of symptoms of 43 months prior to the time of diagnosis.[21] However, with the advent and refinement of modern neuroimaging techniques, this length of time is expected to decrease.[23,24]

Oligodendroglioma, typically supratentorial, arises in the subcortical white matter of the cerebral hemispheres and may expand or invade the cerebral cortex and leptomeninges.[3,23,24,27] These lesions predominate in the frontal lobes, and as many as $50 \%$ of all oligodendrogliomas are found in this location.[4,22,30,32] The temporal and parietal lobes are also frequently involved, with the occipital lobe being the least common. The regional frequency of oligodendroglioma has been roughly associated with the relative mass of each lobe.[3] Occasionally, oligodendroglioma may be intraventricular in location and has also been reported within the spinal cord.[22] The true incidence of intraventricular lesions may be lower than previously described because many central neurocytomas may have been mistakenly diagnosed as oligodendrogliomas.[3]

The pathological diagnosis of DNT was described by Dumas-Duport, et al.,[7] in 1988. Only a few case reports and small series have since been published on DNT; therefore, relatively little is known about the natural history of this entity. However, a review of these series (Table 1) has yielded information regarding the natural history of this lesion.[1,7,9,12,15,25,26,28,31] The true incidence of DNT is unknown; however, since its recognition, only 91 cases have been reported, suggesting that this lesion is quite uncommon, probably representing fewer than $1 \%$ of primary intracranial neoplasms. There is a slight male preponderance, with males being affected $58.2 \%$ of the time. The incidence of DNT, in contrast to oligodendrogliomas, appears to be higher in patients in a younger age group, with $47 \%$ of the reported cases in patients younger than 10 years of age; $28 \%$ in patients between 10 and 20 years of age; and only $20.8 \%$ in patients older than 20 years of age. However, numerous reports indicate the diagnosis of DNT may not be discerned until the fourth decade of life. $[9,14,17]$

\begin{tabular}{|c|c|c|c|c|c|c|c|c|c|c|c|c|c|}
\hline \multirow[b]{3}{*}{ Authors \& Year } & \multirow{3}{*}{$\begin{array}{l}\text { No. of } \\
\text { Cases }\end{array}$} & \multicolumn{4}{|c|}{$\begin{array}{c}\text { TABLE } \\
\text { OF CL NICAL FE }\end{array}$} & ATURES & N PAT & ENTS $W$ & $\mathrm{HCl}$ & & & & \\
\hline & & & & \multirow{2}{*}{$\begin{array}{l}\text { Sej- } \\
\text { zures }\end{array}$} & \multirow{2}{*}{$\begin{array}{l}\mathrm{H} A \mathrm{I} \\
\mathrm{ICP}\end{array}$} & \multirow{2}{*}{$\begin{array}{l}\text { Neuro- } \\
\text { logical } \\
\text { Deficit }\end{array}$} & \multicolumn{4}{|c|}{ Location of Tumor } & \multicolumn{3}{|c|}{$\begin{array}{c}\text { Age [yrs] } \\
\text { at Diagnosis }\end{array}$} \\
\hline & & $\mathrm{M}$ & $\mathrm{F}$ & & & & $\mathrm{Fr}$ & Temp & Par & Occ & $<10$ & $10-20$ & $>20$ \\
\hline Dumas-Du port, et al., 1988 & 39 & 23 & 16 & 39 & 3 & 2 & 12 & 24 & 2 & 1 & 23 & 16 & - \\
\hline Koeller \& Dillon, 1992 & 6 & 4 & 2 & 6 & - & - & - & 5 & - & 1 & 1 & 2 & 3 \\
\hline Prayson \& Estes, 1992 & 2 & 0 & 2 & 2 & - & - & - & 2 & - & - & 1 & 1 & - \\
\hline Kuroi ma, et al., 1994 & 10 & 5 & 5 & 10 & - & - & 4 & 4 & 2 & - & 1 & 4 & 5 \\
\hline Raymond, et al., 1994 & 16 & 9 & 7 & 15 & 1 & 1 & 1 & 15 & - & - & 5 & 3 & 8 \\
\hline Abe, et al., 1995 & 3 & 2 & 1 & 3 & - & - & 1 & 2 & - & - & 1 & - & 2 \\
\hline Imanga, et al., 1995 & 1 & - & 1 & 1 & - & - & - & 1 & - & - & - & - & 1 \\
\hline Taratuto, et al., 1995 & 14 & 10 & 4 & 14 & - & - & 6 & 6 & 1 & 1 & 11 & 2 & - \\
\hline $\begin{array}{l}\text { Total } \\
(\%)\end{array}$ & 91 & 53 & 38 & 90 & 4 & 3 & 24 & 59 & 5 & 3 & 43 & 28 & $\begin{array}{r}19 \\
(21)\end{array}$ \\
\hline
\end{tabular}

With one exception,[26] all reported cases of DNT were associated with seizure activity as the presenting 
feature. Typically, seizures are of the complex partial type and may be refractory to anticonvulsant medication. Complaints of headache or signs of increased intracranial pressure, such as papilledema, were found in only $4.3 \%$ of patients with DNT. Additionally, the presence of a neurological deficit was found in only three patients $(3.3 \%)$ and represented quadrantanopsias or dysphasia, all of which were directly attributable to the location of the lesion.[7,26] All reported cases of DNT have been found in the supratentorial region and have involved the cerebral cortex and subcortical white matter. Although DNT is typically a solitary lesion, there have been two cases of multifocal lesions with involvement of deep nuclear structures.[17] The majority of cases are found in the temporal lobe (64.8\%), followed by the frontal lobe (26.4\%), parietal lobe (5.4\%), and, rarely, the occipital lobe (3.3\%).

\section{Neuroimaging Studies}

The imaging characteristics of oligodendroglioma and DNT on computerized tomography (CT) and MR imaging are strikingly similar and often indistinguishable. Notably, both lesions tend to occupy the cortex and subcortical white matter in the supratentorial compartment. However, DNT has a predilection for the temporal lobe and oligodendroglioma favors the frontal lobe. Noncontrast-enhanced CT scans show well-demarcated lesions that are hypodense to surrounding brain. $[6,7,23,24]$ Evidence of intratumoral calcification is present in as many as $23 \%$ of DNTs[ $[7,14]$ and in as many as $50 \%$ of oligodendrogliomas.[16,19,21] Evidence of the lesion's long-standing nature, such as calvarial erosion or enlargement of the temporal fossa, occurs in $30 \%$ of DNTs and $17 \%$ of oligodendrogliomas. $[7,16]$ The presence of intratumoral hemorrhage may be found in oligodendrogliomas; however, this finding has not been described in DNTs.

Routine MR imaging sequences reveal both lesions to be well demarcated, hypointense on $\mathrm{T}_{1}$-weighted images, and hyperintense on $\mathrm{T}_{2}$-weighted images (Figs. 1 and 2).[6,10,12,14-16,19] Magnetic resonance imaging is superior to CT scanning for discerning soft-tissue detail and more sensitive for revealing cystic changes, the presence of edema, or contrast enhancement. Both entities demonstrate cystic changes occurring in 50\% of DNTs and 23\% of oligodendrogliomas.[7,16] Additionally, both lesions show enhancement with contrast administration in less than $50 \%$ of cases.[6,7,12,14-16,19,23,24] The presence of edema on $\mathrm{T}_{2}$-weighted images is conspicuously absent in DNTs; however, it is only present in approximately $30 \%$ of oligodendrogliomas.[16] Single-photon emission CT has been used in limited fashion with DNT, and this shows hypoperfusion or poor isotope uptake, similar to low-grade glioma.[1]

A recently developed technique, MR spectroscopy allows the determination of certain biochemical properties of the brain in vivo. Specifically, a volume of interest is selected, and certain mobile tissue metabolites may be detected and quantified on a spectral array. Certain distinguishable characteristics between normal brain and brain tumors have been described.[2,5] Typically, neoplasms show decreased concentrations of $\mathrm{N}$-acetyl-aspartate (NAA) and creatine/phosphocreatine, whereas choline-containing compounds are increased (Fig. 1 lower right).[2,5] The presence of lactic acid may also be of value in differentiating normal from neoplastic tissue.[2,5] Although the ability to discern individual tumor types on the basis of MR spectroscopy is promising, it remains inconclusive.

The spectral array pattern for DNT has not been previously described. The MR spectroscopy performed in our patient with DNT (Case 2) provided a spectral array pattern distinctly different from the oligodendroglioma and from that previously described for intracranial neoplasms.[2,5] Interestingly, there was no suppression of the NAA or creatine peaks, and there was no increase in the choline concentration (Fig. 3 lower right). Although this is the first report to describe the use of MR spectroscopy in DNT, the marked differences compared with the spectral array patterns of other neoplasms indicates that this technique may prove to be a useful tool in the evaluation of patients with intracranial masses who present with seizures. 
The ability to establish the diagnosis with MR spectroscopy could obviate the need for surgical intervention and affect the therapeutic course for these patients. Further experience with MR spectroscopy is needed to confirm its specificity.

\section{Histopathological Features}

Light microscopy with routine and immunohistochemical staining differentiates DNT from oligodendroglioma because each has certain characteristics. However, misdiagnosis may occur.[7] The histopathological appearance of oligodendroglioma has been well described; however, the diagnosis can be elusive, especially in terms of histological grade, because the differences between pure low-grade and anaplastic oligodendroglioma may be subtle.

Typically, the oligodendroglioma consists of sheets of small round cells that are fairly uniform (Fig. 2). Cellular and nuclear pleomorphism are conspicuously absent.[3,27] The sheets of cells are involved with a network of delicate capillaries that may resemble "chicken wire."[3] On fixed specimen, neoplastic oligodendroglia have a perinuclear halo, as an artifact secondary to delayed fixation, which yields a "fried egg" appearance.[3,27]

Oligodendroglioma may be sharply demarcated from the surrounding normal white matter as evidenced by increased cellularity, or the neoplasm's interface with adjacent white matter may be subtle. These neoplasms typically infiltrate the overlying cortex, making their margins from surrounding normal brain characteristically indistinct (Fig. 2 upper left).[3,27] Perineuronal satellitosis is a characteristic finding in oligodendroglioma. The presence of calcium deposits within the tumor may be seen in 28 to $40 \%$ of cases on preoperative imaging studies; [21,27] however, calcium may be present in as many as $90 \%$ of microscopic examinations.[3] Although not pathognomonic for oligodendroglioma, this is significantly higher than for other glial neoplasms.

Glial fibrillary acidic protein (GFAP) staining may be of some value in characterizing oligodendroglioma. Unlike astrocytic neoplasms, oligodendroglioma do not typically stain for GFAP; however, as many as 50\% of pure oligodendroglioma may stain for GFAP (Fig. 2 lower right), probably owing to the presence of reactive astrocytes.[27] Additionally, if oligodendrogliomas have a significant astroglial component, they are termed mixed oligodendroglioma or oligoastrocytomas. Furthermore, as the amount of anaplasia increases so does the reactivity of GFAP staining.[3,27] Oligodendrogliomas tend to be positive for S-100 protein (Fig. 2 lower left), and they typically stain for Leu 7; however, no immunostain has been developed that is specific for oligodendroglioma.

As with other glial neoplasm, oligodendrogliomas span a spectrum ranging from benign to anaplastic to frankly malignant (represented by glioblastoma).[3] The hallmarks of anaplasia in oligodendroglioma include increasing cellularity, vascular and endothelial proliferation, increased nuclear/cytoplasmic ratio, increasing numbers of mitotic figures, and cellular atypia. However, the overall cellular uniformity is reasonably maintained in malignant oligodendroglioma compared with other malignant glioma.[3]

Histologically, DNT may resemble oligodendroglioma or oligoastrocytoma, but certain histological features of DNT allow differentiation from these other neoplasms. The initial histological diagnostic criteria described for DNT included cortical location, multinodular architecture with the nodular component resembling oligodendroglioma, oligoastrocytomas or astrocytomas, the presence of focal cortical dysplasia, and the presence of a specific glioneuronal element.[7] Subsequent studies and further examination of this entity have resulted in further delineation and revision of the diagnostic criteria.

With the exception of two cases,[17] all reported cases involve the cerebral cortex or the neocortex of the 
temporal lobe. The two exceptions were cases of multifocal involvement in which the affected structures included the basal ganglia, thalamus, and pons. The presence of focal cortical dysplasia is frequently described; however, it is not invariably present and may only be detected in an optimally oriented specimen.[6,7]

The most frequently found and most important features of DNT include multinodular architecture and a specific glioneuronal element. Typically, the multinodular architecture consists of well-circumscribed nodules displaying a high degree of cellular polymorphism. The polymorphic nature is due to the presence of multiple types of cells including astrocytes, neurons, and oligodendroglia (Fig. 4 upper left and right). Within these nodular foci, a wide range of cellular pleomorphism, ranging from bizarre to mature, may be found. $[7,25,26]$ Mitoses may be encountered within these nodules but are characteristically few in number.[6,7,17,25,26] A glomeruloid capillary network of proliferating, delicate capillaries may also be encountered, but true endothelial proliferation is not present.[6,7] Necrosis, although rarely described, may also be present.[6] Unlike glioma, however, the characteristics of cellular atypia, vascular prominence, and necrosis are not indicators of malignancy in DNT. Furthermore, in contrast to oligodendroglioma, perineuronal satellitosis is not a feature in DNT.

The last hallmark feature seen in DNT is the specific glioneuronal element, which is characterized by an area of abnormality that typically replaces the cortex. It consists of either compact or alveolar architecture within an area of extracellular mucinous accumulation in which rows of cells and capillaries are oriented perpendicular to the cortex.[6,7,17] The cells lining these capillaries are most commonly oligodendroglia, but astrocytes and neurons may also be present. These cells usually test positive for S-100 protein and may occasionally stain for GFAP.[8,17,31] Within the glioneuronal element, neurons appear to be free floating within a sea of surrounding mucin (Fig. 4 lower left and right).[6,7] The presence of the specific glioneuronal element confirms the diagnosis of DNT even if the other features are absent; [6] however, the absence of this element does not exclude the diagnosis because it is not present in all cases.[7,9] In fact, it has been proposed that some lesions without any of the specific DNT characteristics, which appear histologically to be low-grade gliomas but behave clinically as DNTs, should in fact be classified as DNTs.[6]

\section{Therapy and Outcome}

As indicated by the natural history of these entities, the clinical course, appropriate therapy, and survival of patients with DNT or oligodendroglioma differ significantly. Oligodendrogliomas are true neoplasms that, if left untreated, will proliferate, and ultimately the patient will succumb to their disease. The median duration of disease from the onset of symptoms until the time of death in untreated patients is 14 months,[21] which increases to as much as 9.8 years with treatment.[29] Traditionally, the treatment of choice for oligodendroglioma has been surgical resection followed by radiation therapy. Compared with untreated patients, surgical resection resulted in a fivefold increase in survival from the onset of symptoms.[21] The 5 -year survival rate ranges from 34 to $66 \%$ and the 10-year survival rate from 24 to $34 \%$ in patients undergoing surgical resection with or without adjuvant therapy.[4,13,21,22,30] Many factors, including histological grade, degree of surgical resection, neurological status, and ABO blood group have been shown to correlate with prognosis. $[4,21,22,30]$ Additional treatment with postoperative radiation therapy has yielded equivocal results: one study has shown that radiation therapy correlated with a favorable outcome;[4] however, three series have shown that adjuvant radiation therapy had no significant impact on survival compared to patients treated with surgery alone.[21,22,30] Recently, studies suggest a significant benefit of chemotherapy in the treatment of anaplastic oligodendroglioma.[18,20]

In contrast to oligodendroglioma, DNTs have a benign course. Treatment for DNT is surgical resection; however, there is no cohort of untreated patients to serve as controls. There has been no report of 
postoperative radiographic or clinical progression of disease, even in patients who underwent subtotal resection. $[1,6,7,9,12,15,25,26,31]$ In fact it has been recommended that surgery is indicated in patients with DNT only for the treatment of medically intractable seizures because of the tumor's extremely benign clinical course.[6] However, as has been previously outlined, it is often impossible preoperatively to differentiate DNT from low-grade glioma, especially oligodendroglioma. Magnetic resonance spectroscopy may improve the ability to diagnose DNT preoperatively, thus potentially altering the course of therapy in those patients without intractable epilepsy.

\section{CONCLUSIONS}

Dysembryoplastic neuroepithelial tumor is a recently described neoplasm with a benign clinical course that typically presents with intractable epilepsy of the complex partial type. Although these lesions tend to occur in younger patients, DNTs may be clinically and radiographically indistinguishable from low-grade glial neoplasms, especially oligodendrogliomas. Our two cases illustrate the potential use of MR spectroscopy in the evaluation of these patients. The MR spectroscopic pattern of DNT has not been previously reported; however, this report indicates that the pattern may be distinctly different from glial neoplasms. The potential advantage of this tool may be its ability to accurately diagnose these lesions in vivo and thereby alter the course of treatment; however, further studies using this technique are necessary. Dysembryoplastic neuroepithelial tumor must be included in the differential diagnosis of cortically located lesions in patients presenting with seizures.

\section{References}

1. Abe M, Tabuchi K, Tsuji T, et al: Dysembryoplastic neuroepithelial tumor: report of three cases. Surg Neurol 43:240-245, 1995

2. Bruhn H, Frahm J, Gyngell ML, et al: Noninvasive differentiation of tumors with use of localized H-1 MR spectroscopy in vivo: initial experience in patients with cerebral tumors. Radiology 172:541-548, 1989

3. Burger PC, Scheitauer BW, Vogel FS: Surgical Pathology of the Nervous System and its Coverings. New York: Churchill-Livingstone, 1991, pp 306-324

4. Celli P, Nofrone I, Palma L, et al: Cerebral oligodendroglioma: prognostic factors and life history. Neurosurgery 35:1018-1035, 1994

5. Demaerel P, Johannik K, Van Hecke P, et al: Localized 1H NMR spectroscopy in fifty cases of newly diagnosed intracranial tumors. J Comput Assist Tomogr 15:67-76, 1991

6. Dumas-Duport C: Dysembryoplastic neuroepithelial tumours. Brain Pathol 3:283-295, 1993

7. Dumas-Duport C, Scheithauer BW, Chodkiewicz JP, et al: Dysembryoplastic neuroepithelial tumor: a surgically curable tumor of young patients with intractable partial seizures. Report of thirty-nine cases.

Neurosurgery 23:545-556, 1988

8. Hirose T, Scheithauer BW, Lopes MB, et al: Dysembryoplastic neuroepithelial tumor (DNT): an immunohistochemical and ultrastructural study. J Neuropathol Exp Neurol 53:184-195, 1994

9. Iwanaga K, Takahashi H, Kameyama S, et al: Dysembryoplastic neuroepithelial tumor: report of a case without typical glioneuronal elements. Acta Neuropathol 89:284-289, 1995

10. Kimura S, Kobayashi T, Hara M: A case of dysembryoplastic neuroepithelial tumor of the parietal lobe with characteristic magnetic resonance imaging. Acta Paediatr Jpn 38:168-171, 1996 
11. Kleihues P, Burger PC, Scheithauer BW: The new WHO classification of brain tumours. Brain Pathol 3:255-268, 1993

12. Koeller KK, Dillon WP: Dysembryoplastic neuroepithelial tumors: MR appearance. AJNR 13:1319-1325, 1992

13. Kros JM: Oligodendrogliomas: clinicopathological correlations. J Neurooncol 24:29-31, 1995

14. Kuroiwa T, Bergey GK, Rothman MI, et al: Radiologic appearance of the dysembryoplastic neuroepithelial tumor. Radiology 197:233-238, 1995

15. Kuroiwa T, Kishikawa T, Kato A, et al: Dysembryoplastic neuroepithelial tumors: MR findings. J Comput Assist Tomogr 18:352-356, 1994

16. Lee YY, Van Tassel P: Intracranial oligodendroglioma: imaging findings in 35 untreated cases. AJR 152:361-369, 1989

17. Leung SY, Gwi E, Ng HK, et al: Dysembryoplastic neuroepithelial tumor: a tumor with small neuronal cells resembling oligodendroglioma. Am J Surg Pathol 18:604-614, 1994

18. Macdonald DR: New therapies of primary CNS lymphomas and oligodendrogliomas. J Neurooncol 24:97-101, 1995

19. Margain D, Peretti-Viton P, Perez-Castillo AM, et al: Oligodendrogliomas. J Neuroradiol 18:153-160, 1991

20. Mason WP, Krol GS, DeAngelis LM: Low-grade oligodendroglioma responds to chemotherapy.

Neurology 46:203-207, 1996

21. Mork SJ, Lindegaard KF, Halvorsen TB, et al: Oligodendroglioma: incidence and biological behavior in a defined population. J Neurosurg 63:881-889, 1985

22. Nijjar TS, Simpson WJ, Gadalla T, et al: Oligodendroglioma: the Princess Margaret Hospital experience. Cancer 71:4002-4006, 1993

23. Peterson K, Cairncross J G: Oligodendroglioma. Cancer Invest 14:243-251, 1996

24. Peterson K, Cairncross JG: Oligodendrogliomas. Neurol Clin 13:861-873, 1995

25. Prayson RA, Estes ML: Dysembryoplastic neuroepithelial tumor. Am J Clin Pathol 97:398-401, 1992

26. Raymond AA, Halpin SFS, Alsanjari N, et al: Dysembryoplastic neuroepithelial tumor. Features in 16 patients. Brain 117:461-475, 1994

27. Russell DS, Rubenstein LJ: Pathology of Tumors of the Nervous System, ed 5. London: Edward Arnold, 1989, pp 172-187

28. Scott RM, di Rocco C, Canady A, et al: How would you treat this patient? Dysembryoplastic neuroepithelial tumor. Surg Neurol 46:23-27, 1996

29. Shaw EG, Scheithauer BW, O'Fallon JR: Supratentorial gliomas: a comparative study by grade and histologic type. J Neurooncol 31:273-278, 1997

30. Shaw EG, Scheitauer BW, O'Fallon JR, et al: Oligodendrogliomas: the Mayo Clinic experience. J 
Neurosurg 76:428-434, 1992

31. Taratuto AL, Pomata H, Sevlever G, et al: Dysembryoplastic neuroepithelial tumor: morphological, immunocytochemical, and deoxyribonucleic acid analyses in a pediatric series. Neurosurgery 36:474-481, 1995

32. Whittle IR, Beaumont A: Seizures in patients with supratentorial oligodendroglial tumours. Clinicopathological features and management considerations. Acta Neurochir 135:19-24, 1995

Manuscript received January 28, 1998.

Accepted in final form March 23, 1998.

Address reprint requests to: Keith M. Rich, M.D., Department of Neurological Surgery, Washington University School of Medicine, Campus Box 8057, St. Louis, Missouri 63110. 